\title{
EFFICACY OF MULTIDETECTOR COMPUTED TOMOGRAPHY IN QUANTITATIVE AND QUALITATIVE ANALYSIS OF FACIAL SYMMETRY AFTER ZYGOMATICOMAXILLARY COMPLEX FRACTURES MANAGEMENT
}

\author{
Abdel Aziz Baiomy Abdullah Baiomy* and Ibraheem Mahmoud Mwafey ***
}

\begin{abstract}
Aim : The aim of present study was quantitative and qualitative analysis of facial symmetry after ZMC fractures management using multidetector computed tomography .

Patients and methods: A descriptive study of consecutive 40 adult patients with recent isolated unilateral ZMC fractures who were otherwise healthy was performed from 2019 through 2020. Patient demographics, and both clinical, and CT radiographical assessments were done preoperatively and postoperatively to evaluate postoperative complications, patient satisfaction score, and quality and quantity of facial symmetry.
\end{abstract}

Results: A total 16 patients complained postoperative complications. Postoperative facial asymmetry was observed in $22.5 \%$ of patients . The patient satisfaction score gradually increased at all intervals of study. As for the pre- and post-operative radiographic analysis; a total of 28 fractures were considered acceptably reduced, while 12 fractures was considered sub-optimally reduced. There was highly significant difference between preoperative and postoperative values of facial asymmetry indexes $(\mathrm{p} \leq 0.01)$

Conclusion: Our results demonstrated that multidetector computed tomography can be used as accurate imaging modality for quantitative and qualitative analysis of facial symmetry after ZMC fractures management leading to improve postoperative esthetic outcome .

KEYWORDS: ZMC fracture, facial symmetry, rigid internal fixation, multi-detector computed tomography, midface fracture.

* Assistant Professor of Oral and Maxillofacial Surgery, Faculty of Dental Medicine, Al-Azhar University, (Assiut, Boys), Assiut, Egypt.

** Lecturer of Oral Medicine, Periodontology, Oral Diagnosis and Dental Radiology Faculty of Dental Medicine Al-Azhar University, (Assiut, Boys) Assiut, Egypt. 


\section{INTRODUCTION}

Restoring of the zygomaticomaxillary complex (ZMC) to 3 dimensional (3D) original position after fracture management has been a constant challenge for maxillofacial surgeons because of its important position in facial esthetics. The ZMC is corner stone in defining facial aesthetics due to its prominence and convex shape. It also serves as major functional buttress of the face. The paired zygomas have surgical importance in restoring lost orbital volume and they serve as references in restoring facial symmetry during midface fractures reduction ${ }^{(1,2)}$.

Usually, ZMC fractures clinically associated with face deformity due to increased facial width after loss of cheek projection. Therefore, primary goals are both restoration of facial projection \& symmetry and restoration of orbital volume \& globe position ${ }^{(3,4)}$. Management of ZMC fractures may ranges from no intervention and observation to open reduction and internal fixation of at least three of the zygoma's four articulations. But views differ sharply as to the best one ${ }^{(5-7)}$.

Several studies ${ }^{(8-10)}$ have been performed to evaluate different modalities of diagnosis, analysis, surgical management, and postoperative complications assessment of ZMC fractures. However, it is difficult to be sure about adequate reduction and patient satisfaction .

Recently, multi-detector computed tomography (MDCT) is one of the most important imaging tools in evaluation of patients with maxillofacial trau$\mathrm{ma}^{(11)}$. Where, 3D reconstruction and multiplanar reformation in coronal and sagittal planes are extremely helpful in assessing the bony architecture in large comminuted, displaced and complex fractures involving multiple planes that helps the surgeons for appropriate planning and may aid in proper reduction of fractures in the three-dimensions of space to assure proper facial symmetry ${ }^{(12,13)}$. One of the most important landmark to assess postoperative facial symmetry is malar eminence. It defined as the most prominent point of the malar bone on axial CT slices of the ZMC, is a key point for facial symmetry, and its inadequate restoration is the most common error in ZMC fracture treatments ${ }^{(7)}$.

There were few studies ${ }^{(14,15)}$ have been conducted based on quantitative and qualitative analysis of postoperative symmetry after ZMC reduction as tool to avoid postoperative inadequate restoration leading to proper patient satisfaction. Therefore, the aim of current study was evaluation efficacy of MDCT as adjunctive tool to assess quality of bone reduction and quantify postoperative facial symmetry after ZMC management.

\section{PATIENTS AND METHODS}

This study is a prospective descriptive study carried out on a total number of forty patients. All patients enrolled were suffering from unilateral moderate-energy OZC fractures. The study conducted at the Department of Oral and Maxillofacial Surgery, Faculty of Dentistry, AlAzhar university-Assuit branch, Egypt over one years (from April 2019 to April 2020). Patients signed informed consent after discussing all information about the surgery. The study was approved by the Al-Azhar University ethical committee and done following the Helsinki declaration of human research conduct. The observer were not blinded at the time of the patient examinations and radiographic evaluation. The patients were not blinded because they were informed aim of study.

\section{Inclusion and exclusion criteria}

Inclusion criteria were unilateral moderateenergy OZC fractures, need OIRF within two weeks after injury, confirmed by preoperative clinical and radiographic evaluation, and medically fit patients who didn't have any contraindication to the surgery. Patients were excluded from the study if they had nondisplaced ZMC fracture, supraorbital rim fracture, inadequate treatment or followup information, a previous ZMC fracture, facial 
asymmetry from causes other than ZMC fracture, and inability to provide $\mathrm{CT}$ images.

\section{Preoperative phase:}

Demographic information (personal history, history of trauma), and the medical history of each patient were documented. Preoperative extraoral and intraoral clinical examination (fig. $1 \mathrm{~A} \& \mathrm{~B}$ ), and radiographic examination included $\mathrm{CT}$ scans in the three planes with 3D reconstruction were done for all patients. All routine laboratory investigations were done for all patients.

\section{Operative Phase}

The operation was performed under general anesthesia with oroendotracheal intubation. The oral cavity was first scrubbed with povidone iodine, then all around extraoral surgical site followed by draping with sterile towels, exposing only the area of surgery. Open reduction and internal fixation (ORIF) was the treatment modality of choice for such injuries, of which 40 patients were treated. All ZMC fractures were fixed by three points fixation technique compined with titanium mesh reconstruction in 12 fractures only (Fig.1C,D\&E), .

\section{Postoperative Phase care}

Analgesics, anti-inflammatory drugs, antibiotics, and antiseptic mouthwash were prescribed for 7 days postoperatively. The maintenance of a soft diet, and good oral hygiene was recommended for 2 weeks after surgery. Patients were clinically followed up, on 3rd day followed by 7 th day for suture removal, later followed up on 1,3 , and 6 months post-operatively.

\section{Postoperative evaluation}

Postoperative clinical, and patient satisfaction assessments were done at 1,3 , and 6 months postoperatively. While, radiographic assessments were done immediately and at two month follow up.

\section{Clinical evaluation}

Post-operative clinical evaluation was conducted through visual inspection of the zygomatic projection from behind the patient, and from in front of the patient to check for obvious facial asymmetry. Also, palpation of the zygomatic complex articulations to inspect for any palpable steps, and palpation of plate prominence over lateral orbital rim and infraorbital rim.

\section{Patient satisfaction score for facial symmetry}

Patient satisfaction was assessed by a gradient scale from 1 to 10,1 being totally unsatisfied and 10 being completely satisfied to facial symmetry outcome after the trauma and the surgical intervention after 1,3 , and 6 months post-operatively

\section{Radiographic evaluation}

CT images were acquired on a SOMATOM Definition Flash scanner (Siemens AG, Muenchen, Germany) following trauma protocols for CT scanning $(512 \times 512$ matrix, $150 \mathrm{~mA}, 140 \mathrm{kV}$, scan time $=2.0$ seconds) the scans were performed at consecutive $1.0 \mathrm{~mm}$ intervals extending from the superior aspect of the orbit to inferior border of the mandible axially and from the anterior nasal spine to the posterior aspect of the foramen magnum coronally. Full CT studies, both pre- and postoperative were transferred to Syngo Acquisition Work Place (Siemens AG, Muenchen, Germany) and displayed in bone algorithms for the measurements using syngo.via software (provided by Siemens AG, Germany).

\section{Quality of reduction (qualitative analysis)}

Each patient's Digital Imaging and Communication Medicine (DICOM) data was transferred to the workstation for a series of measurements as described by Furst et al ${ }^{(13)}$. The coronal and axial midlines were defined and 3 landmarks were identified to determine the posterior zygomatic complex width (measurement 1), anterior zygomatic complex width (measurement 2), zygomatic complex 
projection (measurement 3 ), and zygomatic complex height (measurement 4). Measurements were obtained pre- and postoperatively after two month from surgery to assess the quality of reduction to the nearest $0.1 \mathrm{~mm}$, and bony gaps at fracture lines exceeding $2 \mathrm{~mm}$ being considered suboptimal reduction . Also, by comparing contralateral measurements in patients with unilateral injuries as a reference for assessing quality of reduction (fig.2A\&B).

\section{Asymmetry index ( Quantitative analysis) :}

Protruding difference of zygoma, and malar difference using asymmetry index were measured via preoperative and postoperative 3D CT. The protruding difference of zygoma was compared by measuring the distance from the most prominent point of each zygomatic arch to A line. (A line: a virtual line from the pyriform aperture to the condyle of the mandible). The asymmetry index was calculated using the following formula to compare the difference in prominence of the zygomatic arches as described by Khaqani et $\mathrm{al}^{(15)}$, and Ras et $\mathrm{al}^{(16)}$.

\section{Asymmetry index =}

$$
\sqrt{(\mathrm{Hr}-\mathrm{Hl})^{2}+(\mathrm{Vr}-\mathrm{Vl})^{2}+(\mathrm{Dr}-\mathrm{Dl})^{2}}
$$

Where $\mathrm{Hr}$ is right horizontal length, $\mathrm{Hl}$ is left horizontal length, $\mathrm{Vr}$ is right vertical length, $\mathrm{Vl}$ is left vertical length, Dr is right distance from midpoint, and $\mathrm{Dl}$ isleft distance from midpoint.

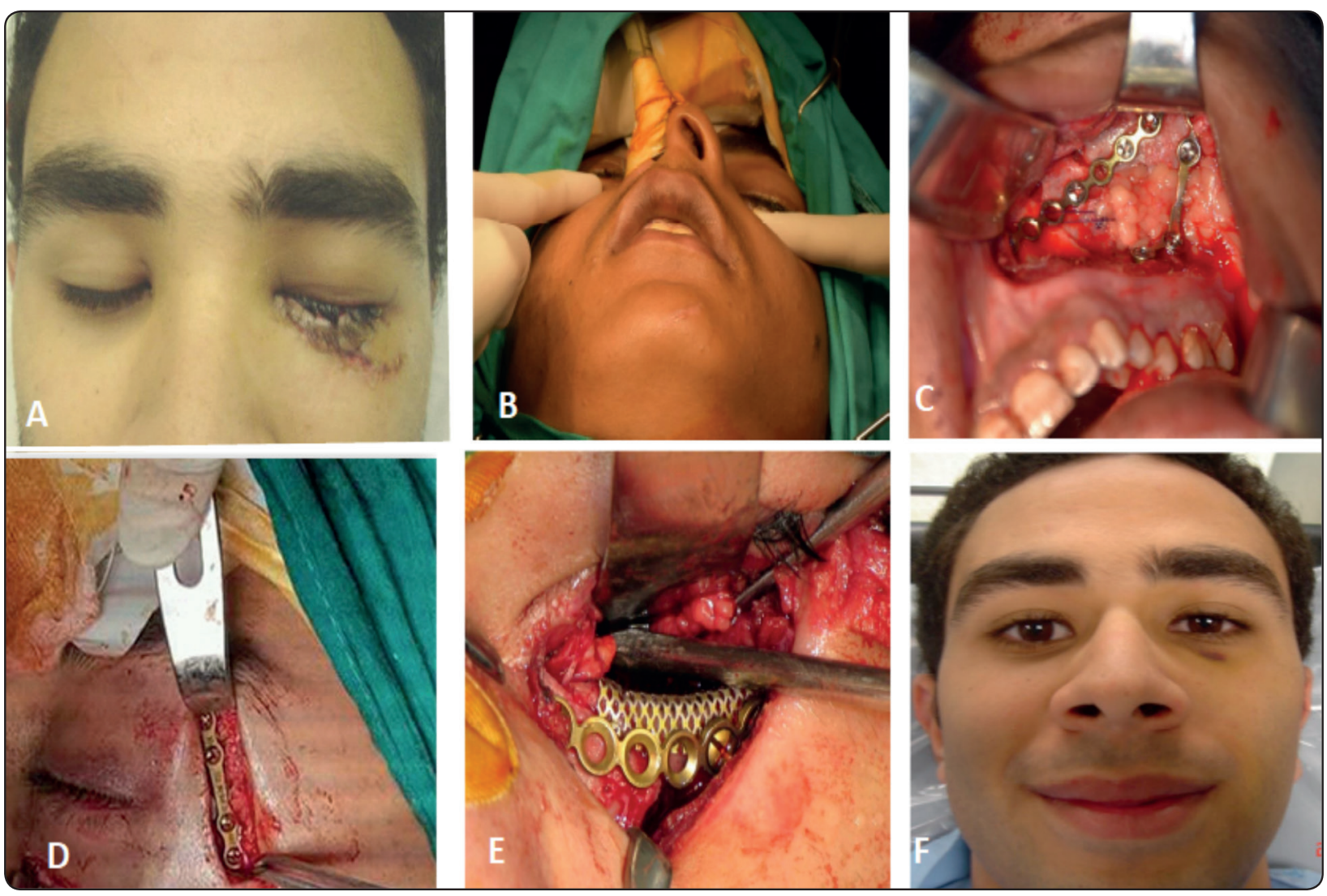

Fig. (1) (A \& B) Preoperative clinical photograph showing facial asymmetry (C) Reduction and fixation of zygomatico maxillary buttress with titanium miniplate (D) Reduction and fixation of frontozygomatic suture by titanium miniplate (E) Reduction and fixation of left inferior orbital rim fracture with titanium miniplate and screws and orbital floor reconstruction with titanium mesh.(F) Postoperative clinical photography showing facial symmetry after bone reduction and fixation of ZMC fracture. 


\section{Statistical analysis}

The study variables data were collected and coded using the statistical package of social sciences (SPSS, version 24, Chicago, Illinois, USA) program for statistical analysis. Descriptive and inferential analyses were performed using t-test and chi-square test for association. P-value was set at or below 0.05 to define significance.

\section{RESULTS}

\section{Demographic data:}

Forty patients (28 male \& 12 female) suffered from unilateral moderate-energy OZC fractures (18 right side and 22 left side) were included in the present study and their age ranged between 23 and 47 years ( mean age $35 \pm 12$ ). Males predominated with a percentage of $70 \%$ of the cases. The result of etiology showed that overall $21(52.5 \%)$ patients had fracture due to RTA, $12(30.0 \%)$ due to fall, $5(12.5 \%)$ due to assault, and $2(5.0 \%)$ due to sports. Concomitant injuries were presented in 13 patients only. Open reduction and internal fixation (ORIF) was the treatment modality of choice for such injuries, of which 28 patients were treated. While, 12 fractures were treated with titanium mesh reconstruction.

\section{Clinical results:}

All fractures healed uneventfully without complications as infection or plate loosening. The hypertrophic scar was seen as late manifestation of wound healing in 7 cases $(17.5 \%)$, where plastic revision was done, and silicone creams or patches were initiated (Fig.1 f).

\section{Postoperative facial asymmetry :}

A total 16 patients (40\% of patients) complained postoperative complications. Facial asymmetry was observed in 9 patients ( $22.5 \%$ ) shared about $65.25 .5 \%$ of total postoperative complications. Other postoperative completions such as flattening of malar prominence was showed in 5 patients (12.5\%), palpable ZMC articulations which occurred in 16 patients (40\%) and resolved in 9 patients after 6 months, abnormal ocular movement occurred in 11 patients (27.5\%) and resolved in 8 patients after one month, post-operative trismus was seen in 13 patients $(32.5 \%)$ and resolved in all of them, 9 patients $(17.5 \%)$ experienced post-operative malocclusion, and 3 patients $(7.5 \%)$ complained from uneven globe position. There was an inverse proportional relationship between the number of complications and the number of patients (table 1).

TABLE (1) : Post-operative clinical outcome of ZMC patients.

\begin{tabular}{|l|c|c|}
\hline Clinical Parameter & $\begin{array}{c}\text { Number of } \\
\text { patients }\end{array}$ & Percent \\
\hline 1-Flattening of malar prominence & 5 & $12.5 \%$ \\
\hline 2-Uneven globe position & 3 & $7.5 \%$ \\
\hline 3-Facial asymmetry & 9 & $22.5 \%$ \\
\hline 4-Abnormal ocular movement & 11 & $27.5 \%$ \\
\hline 5-Palpation of ZMC articulations & 16 & $40 \%$ \\
\hline 6-Malocclusion & 9 & $22.5 \%$ \\
\hline 7-Post-operative trismus & 13 & $32.5 \%$ \\
\hline
\end{tabular}

Patient satisfaction score regarding facial symmetry: Comparing the patient satisfaction score by time, a gradual increase in score was noted in all cases. However, a lower score was noted in cases complained facial asymmetry in comparison to cases complained other complications at all intervals. Unpaired t test revealed that this difference was statistically significant $(\mathrm{p}=0.0072 ; 0.0029 ; 0.0010$ at 1,3 and 6 months respectively).

\section{Correlation between patient satisfaction score and type of complication ( $s$ ):}

It was noted that the patients' opinions about the outcome of the treatment is not standard. Where, Some patients were sub-optimally reduced according to radiographic analysis and did not suffered from facial asymmetry, yet scored higher than 
others whom had apparent facial asymmetry and were acceptably reduced according to radiographs. Trauma patients may accept some postoperative complications such as slightly obvious facial asymmetry or palpable articulations but would not tolerate functional post-operative complications such as abnormal ocular movements or uneven globe position. On the contrary some patients especially female patients would tolerate functional disabilities to a degree but regard their aesthetics highly.

\section{Postoperative Radiographic measurements}

According to post-operative radiographic findings of our study, all radiographic measurements revealed statistically significant differences than preoperative measurements. Postoperatively, there was statistically significant differences between measurements of unaffected side and affected side (fig.2 C\&D).

Regarding quality of bone reduction, a total of $28(70 \%)$ patients were acceptably reduced with a mean difference $(1.24 \pm 0.45)$ and $12(30 \%)$ patients were considered sub optimally reduced with a mean difference of $(3.14 \pm 0.15)$. There was direct proportional relationship between the number of patients with acceptable bone reduction and the number of patients with facial symmetry (table 2) .

Means and standard deviations of protrudi ng difference of zygoma, and facial asymmetry index postoperatively were $1.21 \pm 1.11$, and $1.96 \pm 0.94$ respectively with highly significant difference between before and after surgery measurements ( $\mathrm{p}$ $\leq 0.01)($ table 3$)$.
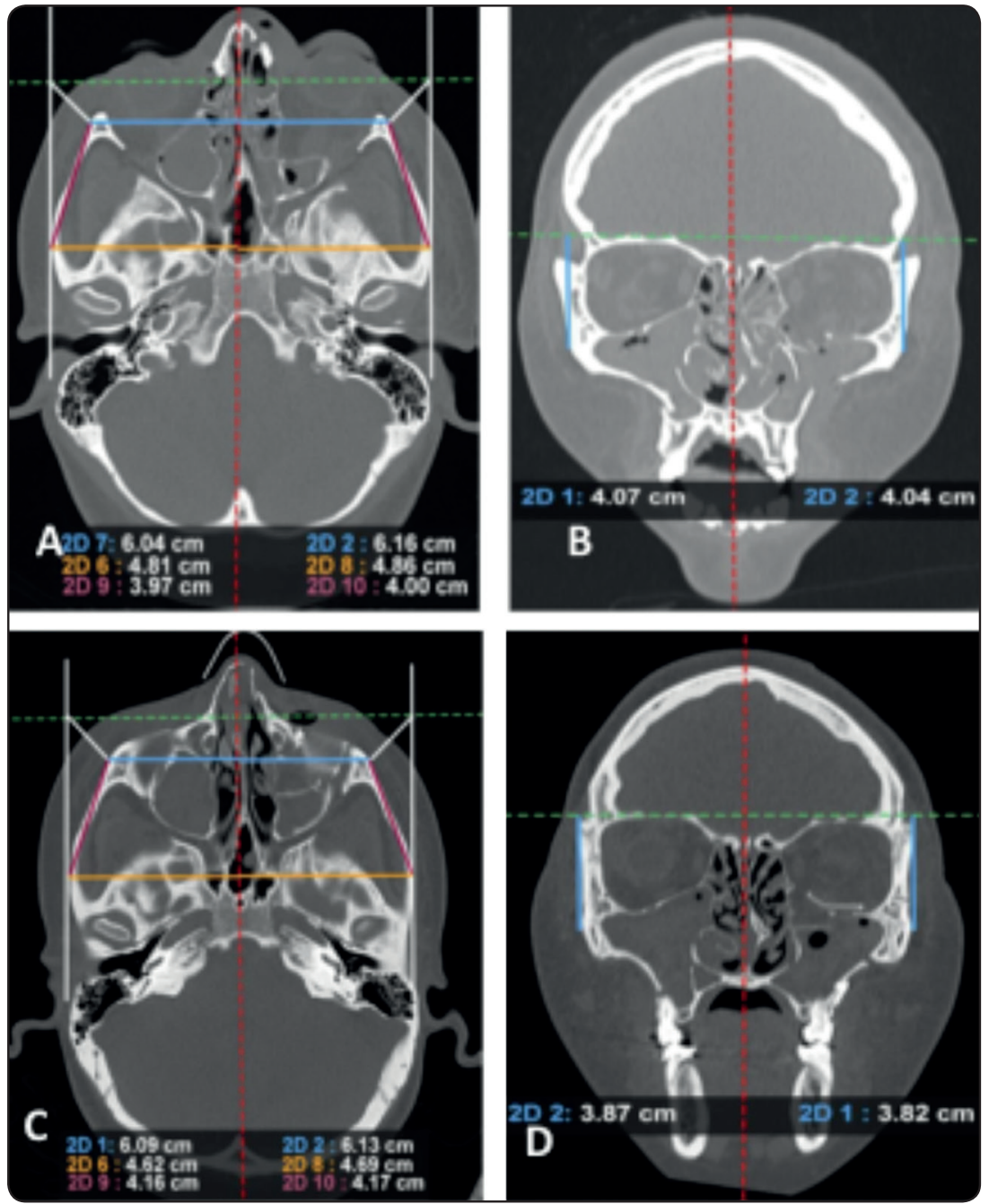

Fig. (2) (A, B) Pre-operative axial and coronal cuts of CT scan showing different measurements. (C, D) Postoperative axial and coronal cuts of CT scan showing different measurements 
TABLE (2) Quality of reduction and mean difference $(\mathrm{mm})$ between affected and unaffected sides

\begin{tabular}{|l|c|c|c|c|}
\hline & $\begin{array}{c}\text { Acceptable } \\
\text { reduction }\end{array}$ & $\begin{array}{c}\text { Suboptimal } \\
\text { reduction }\end{array}$ & t & p \\
\hline No. of cases & 28 & 12 & Chi square $=24.4$ & $0.032^{*}$ \\
\hline Percent of cases & $70 \%$ & $30 \%$ & 6.143 & $0.0051^{* *}$ \\
\hline Mean difference $(\mathrm{mm})$ between right and left sides & $1.24 \pm 0.45$ & $3.14 \pm 0.15$ & 6 \\
\hline
\end{tabular}

* Statistical significance when $p \leq 0.05$

** High statistically significant when $p \leq 0.01$

TABLE (3) Comparison between preoperative and postoperative measurements of affected side

\begin{tabular}{|l|c|c|c|c|}
\hline & Preoperative & Postoperative & t & p \\
\hline ZF displacement & $3.42 \pm 1.26$ & $1.12 \pm 1.23$ & 4.571 & $0.005 * *$ \\
\hline IO displacement & $5.17 \pm 2.97$ & $1.45 \pm 1.64$ & 5.381 & $0.000^{* *}$ \\
\hline Protruding difference of zygoma & $2.96 \pm 2.83$ & $1.21 \pm 1.11$ & 7.8 & $0.041^{*}$ \\
\hline Asymmetry index & $5.72 \pm 1.82$ & $1.96 \pm 0.94$ & 1.9 & $0.000 * *$ \\
\hline
\end{tabular}

* Statistical significance when $p \leq 0.05$

**High statistically significant when $p \leq 0.01$

\section{DISCUSSION}

The zygomatic bone location plays an important role in facial symmetry and esthetics. Therefore, reposition of zygomatic bone to original articulation during ZMC fractures management is essential step to preform esthetics demands and restoration facial symmetry. Numerous studies ${ }^{(8-10)}$ have been published based on analysis of ZMC fractures. However, according our knowledge, few studies ${ }^{(14,15)}$ have been conducted based on quantitative and qualitative analysis of postoperative symmetry after $\mathrm{ZMC}$ reduction.

The aim of the study was quantitative and qualitative analysis of facial symmetry after ZMC fractures management using multidetector computed tomography. The specific aims of this study were to assess the quality of ZMC fracture reduction and to quantify facial symmetry using asymmetry index on postoperative computed tomographic (CT) scans and to determine the relation between patient satisfaction score and quantity of facial symmetry after ZMC reduction.

The mean age of patients in the current study was $35 \pm 12$. Third decade constituted the major group in this study, which is the same as previous studies by Tanaka ${ }^{(17)}$, Anwar et al ${ }^{(18)}$, Fasola ${ }^{(19)}$. This is explained by that the adult is more vulnerable due to dominant outdoor activities at that stage of life as reported by Adekeye ${ }^{(20)}$.

Regards to sex prediction, our study revealed that majority of our patients presenting with OZC fractures were males where $70 \%$ of the cases. This was in the same side with Rana et al ${ }^{(21)}$ explanation. Who explained that females are more confined to indoor activities whereas males are more exposed to external environment during commuting as well as during their jobs. Causes of fractures in our study were variable where $21(52.5 \%)$ patients had fracture due to RTA, $12(30.0 \%)$ due to fall, $5(12.5 \%)$ due to assault, and $5(5.0 \%)$ due to sports. 
This was in accordance with Tanaka et $\mathrm{al}^{(17)}$ and Anwar ${ }^{(18)}$ reports .

Regards to clinical assessment of facial symmetry, our study showed 9 patients with facial asymmetry (about $22.5 \%$ of total postoperative complications). patients satisfaction scores showed lower values in cases complained facial asymmetry in comparison to cases complained other complications at all intervals. Although, patients satisfaction scores about facial symmetry increased by time in these cases.

In our study, immediate postoperative MDCT scanning was preformed immediately at 2 month follow up to evaluate quality of reduction and quantitative analysis of facial symmetry. This was in accordance with Gaziri et al ${ }^{(24)}$ study who compared immediate postoperative CT scans with those obtained after 5 weeks and found that no relevant displacement occurred when adequate fixation was provided. In the same time this was in contrast with Khaqani et al ${ }^{(15)}$ study who reported that immediate postoperative CT scanning was probably sufficient for the assessment of malar bone reduction adequacy.

Our results of radiographic evaluation for reduction quality demonstrated that there are direct relation between facial asymmetry and inadequate reduction. Where mean difference between right and left sides increased in cases with suboptimal bone reduction $(3.14 \pm 0.15 \mathrm{~mm})$ more than in the cases with acceptable reduction (1.24 \pm 0.45$)$. This was matched with Haggerty et al ${ }^{(25)}$ who reported that esthetic deformities after ZMC fracture management occur as a result of inadequate reduction of the fractured components or the development of palpebral fissure deformities. Inappropriate reduction results in a flattening or concavity of the malar area and the lateral and inferior orbital rims.

Pau et $\mathrm{al}^{(26)}$ reported that the mean bilateral difference of the zygomatic prominence in normal adults was less than $2 \mathrm{~mm}$; this was similar to the present results. On the other side, Gong et $\mathrm{al}^{(7)}$ reported that the bilateral difference of zygoma prominence after ZMC reduction and fixation was more than $2.20 \mathrm{~mm}$. This difference between studies can be explained by the use of different landmarks for measuring mediolateral and anteroposterior bilateral differences, and they used a different method for identifying cases of symmetry and asymmetry.

In the current study asymmetry index was used for evaluation quantity of facial asymmetry between affected and unaffected side as in the study by Khaqani et al ${ }^{(15)}$. About $70 \%$ of the present patients had facial symmetry and nearly $30 \%$ of them had marked asymmetry. Values of facial asymmetry indexes decreased postoperatively after reduction and fixation demonstrated proper reduction and fixation . explanation of this, type of fracture was unilateral ZMC feature in our study .This matched with Khaqani et al ${ }^{(15)}$ who reported that the type of ZMC fracture greatly influences the outcome of ZMC fracture treatment. In the same side with the present results, and $\mathrm{Kim}_{\mathrm{im}} \mathrm{al}^{(27)}$ also reported a higher degree of asymmetry for comminuted ZMC fractures

Madkour ${ }^{(28)}$ reported that MDCT is the principle diagnostic imaging tool in maxillofacial trauma. Multiplanar reformatted (MPR) and three-dimensional (3D)-reconstructed images provide a detailed analysis for midfacial fractures and assessment of their severity. This in the same line with our results. Finally, MDCT proved in our study that it had capability to give several accurate measurements. These measurements may be used to evaluate quality of bone reduction and assessment of facial symmetry.

\section{CONCLUSION}

This study demonstrated the valuable role of MDCT in the evaluation of quality of bone reduction and facial symmetry after ZMC fractures. MDCT is time consuming technique which can be used as adjunctive imaging tool for qualitative and 
quantitative analysis of facial symmetry, thus giving better treatment outcome and decrease postoperative facial asymmetry problems .

\section{FUND}

This research did not receive any specific grant from funding agencies in the public, commercial, or not-for-profit sectors.

Conflict of Interest: The authors declare that they have no conflict of interest.

\section{REFERENCES}

1. Salentijn EG, van den Bergh B, Forouzanfar T: A ten-year analysis of midfacial fractures. J Craniofac Surg 41:630, 2013

2. Lee EI, Mohan K, Koshy JC, et al. Optimizing the surgical management of zygomaticomaxillary complex fractures. Semin Plast Surg 2010; 24:389-97.

3. Zou C, Niu F, Liu J, et al: Midface contour change after reduction malarplasty with a modified L-shaped osteotomy: A surgical outcomes study. Aesthet Plast Surg 38:177, 2014

4. Birgfeld CB, Mundinger GS, Gruss JS: Evidence-based medicine: Evaluation and treatment of zygoma fractures. Plast Reconstr Surg 139:168e, 2017

5. Zingg M, Laedrach K, Chen $\mathrm{J}$, et al: Classification and treatment of zygomatic fractures: A review of 1,025 cases. J Oral Maxillofac Surg 50:778, 1992

6. Salentijn EG, Boverhoff J, Heymans MW, et al: The clinical and radiographical characteristics of zygomatic complex fractures: A comparison between the surgically and nonsurgically treated patients. J Craniofac Surg 42:492, 2014

7. Gong X, He Y, An J, et al: Application of a computer-assisted navigation system (CANS) in the delayed treatment of zygomatic fractures: A randomized controlled trial. J Oral Maxillofac Surg 75:1450, 2017

8. Ferrario VF, Sforza C, Ciusa V, et al: The effect of sex and age on facial asymmetry in healthy subjects: A crosssectional study from adolescence to mid-adulthood. J Oral Maxillofac Surg 59: 382, 2001

9. Furst IM, Austin P, Pharoah M, et al: The use of computed tomography to define zygomatic complex position. J Oral Maxillofac Surg 59:647, 2001
10. Peretti N, MacLeod S: Zygomaticomaxillary complex fractures: Diagnosis and treatment. Curr Opin Otolaryngol Head Neck Surg 25:314, 2017

11. Ahmed Madkour NA. Reliability of multidetector computed tomography in rational analysis of midface fractures. $\mathrm{J}$ Oral Maxillofac Radiol 2018;6:21-5.

12. Som PM and Brandwein MS. Facial fractures and postoperative findings. En: Som PM, Curtin HD (eds). Head and neck imaging 2002; Mosby, St. Louis: 374438.

13. Ian Furst, Peter Austin, Michael Pharoh, James Mahony. The use of computed tomography to define zygomatic complex position. J Oral Maxillofac Surg 2001; 59:647654.

14. He Y, Zhang Y, An J, et al: Zygomatic surface markerassisted surgical navigation: A new computer-assisted navigation method for accurate treatment of delayed zygomatic fractures. J Oral Maxillofac Surg 71:2101, 2013

15. M. S. Khaqani, F. Tavosi, M. Gholami, H. R. Eftekharian, and L. Khojastepour, "Analysis of facial symmetry after zygomatic bone fracture management," Journal of Oral and Maxillofacial Surgery, vol. 76, no. 3, pp. 595-604, 2018.

16. F. Ras, L. L. Habets, F. C. van Ginkel, and B. PrahlAndersen, "Method for quantifying facial asymmetry in three dimensions using stereophotogrammetry," the Angle Orthodontist,vol. 65, no. 3, pp. 233-239, 1995.

17. Tanaka ON, Tomitsuka K, Shionoya K, Andou H, Kiimijima Y: Etiology of maxillofacial fractures. Br J Oral Maxillofac Surg 1994, 32:19-23.

18. Anwar BB: Etiology and incidence of maxillofacial fractures in north of Jordan. Oral Surg Oral Med Oral Pathol 1998, 86:31-5.

19. Fasola AO, Obiechina AE, Arotiba JT: Zygomatic complex fractures at the University College Hospital, Ibadan, Nigeria. East Afr Med J 2002, 79:11-3.

20. Adekeye EO: Fracture of zygomatic complex in Nigerian patient. J Oral Surg 1980, 38:596-599.

21. Rana et al:: Surgical treatment of zygomatic bone fracture using two points fixation versus three point fixation-a randomised prospective clinical trial. Trials 2012; 13(36): $1-10$

22. Hwang H-S, Yuan D, Jeong K-H, et al: Three-dimensional soft tissue analysis for the evaluation of facial asymmetry in normal occlusion individuals. Korean J Orthod 42:56, 2012 
23. Nur RB, $\mathrm{C}_{3}$ akan DG, Arun T: Evaluation of facial hard and soft tissue asymmetry using cone-beam computed tomography. Am J Orthod Dentofacial Orthop 149:225, 2016

24. Gaziri DA, Omizollo G, Luchi GHM, et al: Assessment for treatment of tripod fractures of the zygoma with microcompressive screws. J Oral Maxillofac Surg 70:e378, 2012

25. Haggerty CJ., Demian N., Marchena JM., Zygomaticomaxillay Complex Fractures. In: Bagheri S. et al (ed.), Current Therapy in Oral and Maxillofacial Surgery. 2012; 1 st edition, Saunders.
26. Pau CY, Barrera JE, Kwon J, et al: Three-dimensional analysis of zygomatic-maxillary complex fracture patterns. Craniomaxillofac Trauma Reconstr 3:167, 2010

27. Kim DH, Kim RH, Lee J, et al: Evaluation of soft tissue asymmetry using cone-beam computed tomography after open reduction and internal fixation of zygomaticomaxillary complex fracture. J Korean Assoc Oral Maxillofac Surg 40:103, 2014.

28. Ahmed Madkour NA. Reliability of multidetector computed tomography in rational analysis of midface fractures. $\mathrm{J}$ Oral Maxillofac Radiol 2018;6:21-5. 